Pathologe 2021 - 42 (Suppl 2):S197-S198 https://doi.org/10.1007/s00292-021-01010-y Angenommen: 20. September 2021 Online publiziert: 20. Oktober 2021 (c) Springer Medizin Verlag $\mathrm{GmbH}$, ein Teil von Springer Nature 2021

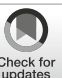

\section{Bericht der AG Molekularpathologie - Virtuelle Pathologietage der DGP 2021}

\author{
Silke Laßmann ${ }^{1}$. Florian Haller ${ }^{2}$. Udo Siebolts ${ }^{3}$ \\ ${ }^{1}$ Institut für Klinische Pathologie, Universitätsklinikum Freiburg, Freiburg im Breisgau, Deutschland \\ ${ }^{2}$ Pathologisches Institut, Universitätsklinikum Erlangen, Erlangen, Deutschland \\ ${ }^{3}$ Institut für Pathologie, Universitätsklinikum Halle, Martin-Luther-Universität Halle, Halle (Saale), \\ Deutschland
}

Auch in diesem Jahr hatte die COVID-19Pandemie die Sprecher und Sprecherin der AG Molekularpathologie wieder seitens digitaler Veranstaltung herausgefordert, was jedoch das zwischenzeitlich virtuell gut trainierte AG-MolPath-Team und das Planungskomitee nicht mehr wirklich überraschen konnte.

Der AG Molekularpathologie wurden als größte Arbeitsgruppe in der DGP 2 Livesitzungen am frühen Donnerstagabend, 10.06.2021, und am Freitag, 11.06.2021, sowie eine begleitende Posterplattform vonseiten des Organisationskomitees zugesprochen. Die Sprecher und Sprecherin sowie der Beirat der AG Molekularpathologie diskutierten im Vorfeld die aktuell brisanten Themen der AG und finalisierten nach Eingang und Bewertung zahlreicher wissenschaftlicher Abstracts $(n=45)$ das virtuelle Programm. Entsprechend hoch war dann auch die Teilnahme an den abendlichen Livesitzungen mit bis zu 150 Zuhörenden und Diskutierenden.

In der ersten Livesitzung wurden innovative Aspekte der Molekularpathologie im Bereich der Liquid-Biopsy-Untersuchungen (ctDNA), der allein tumorbasierten Next-Generation-Sequencing(NGS)Untersuchung, der praxisnahen Übersicht zur Homologen-RekombinationsDefizienz(HRD)-Untersuchung sowie der Übersicht des in 2020 stattgefundenen NGS-Sequenzvarianten-Workshops vorgestellt. Die dabei abgedeckten Technologien umfassten den Avenio-NGSWorkflow für ctDNA und 3 Möglichkeiten der HRD-Testung ("low-pass whole genome sequencing“, "SNP-genotyping array", ampliconbasierte Parallelsequen- zierung - AmoyDx). Für die Bewertung molekularpathologischer Ergebnisse wurden insbesondere die Aspekte der klonalen Hämatopoese bei der Messung von Liquid-Biopsy-Plasmaproben (ctDNA) sowie die zunehmende Relevanz von Keimbahnvarianten in umfassenden NGSPanels aus Tumorproben ohne zusätzliche Analyse des Normalgewebes besprochen. Die Relevanz der Bewertung von molekularpathologischen Ergebnissen wurde abschließend anhand von Beispielen von Sequenzvarianten des NGS-VariantenWorkshops diskutiert. Klar war allen Teilnehmern, dass eine zunehmende inhalttiche Bewertung der rein technologischen Messergebnisse erfolgen muss. Die AGSprecher und -Sprecherin haben den Wunsch der Teilnehmer und Teilnehmerinnen für eine Weiterführung des NGSVarianten-Workshops und sonstiger assoziierter bewertungsrelevanter Aspekte mitgenommen und arbeiten hierzu ein Konzept zusammen mit dem Beirat der AG Molekularpathologie aus.

In der zweiten Livesitzung unter dem Motto "What's New" wurde der Schwerpunkt auf die HRD-Testung gelegt und das Thema mit 3 Vorträgen aus Sicht des Pathologen mit Nutzung des kommerziellen Myriad-Tests (Prof. Dr. C. Denkert, Marburg), aus Sicht der Naturwissenschaftlerin mit Nutzung der verschiedenen Verfahren, wiez. B.via NGS-basiert oder via SNP-genotyping-Arrays (N. Pfarr, TU München), und aus innovativer experimenteller Sicht der Keynote-Sprecherin (Dr. M. Vreswijk, Leiden University Medical Center, NL) durch einen In-situ-Surrogatmarker (RAD51-Loci-Detektion) intensiv beleuchtet. 
Parallel zu den Livesitzungen wurden 17 Posterbeiträge aus der AG Molekularpathologie präsentiert. Diese spannenden Poster reichten von technologischen Beiträgen über einzelne organspezifische molekulare Beiträge bis hin zu experimentellen Forschungsbeiträgen. Das breite Spektrum der Molekularpathologie von Grundlagenforschung über Erprobung und Translation hin zur qualitätsgesicherten molekularpathologischen Diagnostik wurde sehr schön sichtbar.

Als weiterer wichtiger Agendapunkt der AG Molekularpathologie ist noch die im Anschluss an die erste Sitzung am Donnerstag stattgefundene, turnusgemäße Wahl der Sprecher und Sprecherinnen der AG Molekularpathologie sowie deren Beirat in der formalen Mitgliederversammlung zu erwähnen. Nach Ausscheiden von Prof. Dr. med. F. Haller (Erlangen) waren zur Nominierung PD Dr. Dr. U. Siebolts (Sprecher, Halle; 3. Amtszeit) und Prof. Dr. S. Laßmann (Ko-Sprecherin, Freiburg; 3. Amtszeit) sowie als neues Mitglied Dipl.Biol. N. Pfarr (Ko-Sprecherin, TU München; 1. Amtszeit) angemeldet. Dies wurde ohne Gegenstimmen angenommen. An dieser Stelle gilt der Dank der AG Molekularpathologie Herrn Prof. Dr. F. Haller für die geleistete erfolgreiche Arbeit als AG-Sprecher der letzten 4 Jahre. Als Beirat wurden vom neuen Sprecher und Sprecherinnen folgende AG-Mitglieder vorgeschlagen: Prof. Dr. W. Dietmaier (Regensburg), Dr. S. Glombitza (Hannover; neues Beiratsmitglied), Prof. Dr. M. Hummel (Berlin), Prof. Dr. A. Jung (München), Prof. Dr. U. Lehmann (Hannover), Prof. Dr. S. MerkelbachBruse (Köln), Dr. H. Pasternak (Lübeck) und Prof. Dr. A. Stenzinger (Heidelberg). Auch dieses Team wurde ohne Gegenstimmen angenommen. Die Sprecher und Sprecherinnen der AG Molekularpathologie bedanken sich auch im Namen des Beirats herzlich für das Vertrauen und freuen sich auf die zukünftigen gemeinsamen Aufgaben.
In diesem Sinne blicken wir auf sehr gelungene und aktive Virtuelle Pathologietage zurück und freuen uns auf das kommende Jahr in Präsenz in Münster. Das Herbsttreffen der AG Molekularpathologie am 29. und 30. November in Berlin wird uns und allen AG-Mitgliedern und Interessierten die Zeit bis dahin verkürzen.

\section{S. Laßmann, F. Haller, U. Siebolts}

\section{Korrespondenzadresse}

Prof. Dr. Silke Laßmann, BSc., PhD.; M.A.

Institut für Klinische Pathologie, Universitätsklinikum Freiburg

Breisacher Str. 115a, 79106 Freiburg im

Breisgau, Deutschland

silke.lassmann@uniklinik-freiburg.de

PD Dr. med. Dr. nat. med. Udo Siebolts Institut für Pathologie, Universitätsklinikum Halle, Martin-Luther-Universität Halle Postfach 302, 06097 Halle (Saale), Deutschland udo.siebolts@uk-halle.de

\section{Einhaltung ethischer Richtlinien}

Interessenkonflikt. S. Laßmann, F. Haller und U. Siebolts geben an, dass kein Interessenkonflikt besteht.

Für diesen Beitrag wurden von den Autoren keine Studien an Menschen oder Tieren durchgeführt. Für die aufgeführten Studien gelten die jeweils dort angegebenen ethischen Richtlinien.

The supplement containing this article is not sponsored by industry. 